\title{
Possible wormholes in a brane world
}

\author{
K.A. Bronnikov \\ VNIIMS, 3-1 M. Ulyanovoy St., Moscow 117313, Russia; \\ Institute of Gravitation and Cosmology, PFUR, 6 Miklukho-Maklaya St., Moscow 117198, Russid \\ Sung-Won Kim \\ Dept. of Science Education, Ewha Womans University, Seoul 120-750, Kored
}

\begin{abstract}
The condition $R=0$, where $R$ is the four-dimensional scalar curvature, is used for obtaining a large class (with an arbitrary function of $r$ ) of static, spherically symmetric Lorentzian wormhole solutions. The wormholes are globally regular and traversable, can have throats of arbitrary size and can be both symmetric and asymmetric. These solutions may be treated as possible wormhole solutions in a brane world since they satisfy the vacuum Einstein equations on the brane where effective stress-energy is induced by interaction with the bulk gravitational field. Some particular examples are discussed.
\end{abstract}

PACS numbers: $04.50 .+\mathrm{h}: 04.20 . \mathrm{Gz}$

\section{INTRODUCTION}

Lorentzian wormholes as smooth bridges between different universes, or topological handles between remote parts of a single universe, have gained much attention since Morris, Thorne and Yurtsever discussed the connection between wormholes and time machines [1]; see [2, 3] for reviews. It is well known that a wormhole geometry can only appear as a solution to the Einstein equations if the stress-energy tensor (SET) of matter violates the null energy condition (NEC) at least in a neighborhood of the wormhole throat 洵.

Many versions of exotic matter, able to provide NEC violation and to support wormholes, have been suggested. One class of such sources is represented by so-called ghost fields, i.e., fields with explicitly negative energy density, including scalar-tensor theories of gravity with an anomalous sign of the scalar field kinetic term in the Lagrangian [5-13] Another class of static wormholes is obtained with nonminimally coupled scalar fields 6, 14, 15] as a result of conformal continuation [16]. The latter means that a singularity occurring on a certain surface $S$ in the Einstein frame metric, is removed by a conformal mapping to the Jordan frame, and the solution is then continued beyond $S$. It has been shown 16 that a wormhole is a generic result of a conformal continuation if its sufficient conditions are satisfied. In all such cases, however, the two wormhole mouths are located in regions with different signs of the effective gravitational constant. In other words, if one mouth is in a normal gravity region, the other is in an antigravity region [16]. A related problem is the instability of such wormholes caused by the field behavior near the transition surface $S$ [17].

Wormhole solutions have also been obtained in specific versions of dilaton gravity [18] and gravity with torsion

\footnotetext{
*Electronic address: kb@rgs.mccme.ru

$\dagger$ Electronic address: 3 ungwon@mm.ewha.ac.kr
}

[19]. Another approach is to invoke quantum effects, considering wormholes as semiclassical objects [20-23] (see also references therein). In all such cases, NEC violation is probably only possible in extremely strong gravity regions, leading to throat radii close to the Planck length. Wormholes thus seem to be an integral part of the hypothetic space-time foam but their practicability at macroscopic scales still remains vague.

In our view, a natural source of wormhole geometry can be found in the framework of the rapidly developing ideas of brane worlds (24, 25], for reviews see [26]), inspired by the progress in superstring and M-theory [27]. By this concept, the observable world is a kind of domain wall in a multidimensional space (5-dimensional in the simplest case), with large or even infinite extra dimensions. The standard-model fields are confined on the brane while gravity propagates in the surrounding bulk. The gravitational field on the brane itself can be described, at least in models of the type of the second Randall-Sundrum model [25], by the modified 4-dimensional Einstein equations derived by Shiromizu, Maeda and Sasaki [28] from 5 -dimensional gravity with the aid of the Gauss and Codazzi equations. In vacuum, when matter on the brane is absent and the 4-dimensional cosmological constant is zero (a natural assumption for scales much smaller than the size of the Universe), these equations reduce to

$$
G_{\mu \nu}=-E_{\mu \nu}
$$

where $G_{\mu \nu}$ is the 4-dimensional Einstein tensor corresponding to the brane metric $g_{\mu \nu}$ while $E_{\mu \nu}$ is the projection of the 5-dimensional Weyl tensor onto the brane. The traceless tensor $E_{\mu \nu}$ connects gravity on the brane with the bulk geometry (and is sometimes called the tidal SET), so that the set of equations (11) is not closed. Due to its geometric origin, $E_{\mu \nu}$ does not necessarily satisfy the energy conditions applicable to ordinary matter. Thus, examples are known [29] when negative energies on the brane are induced by gravitational waves or black strings in the bulk. Therefore, if the brane world concept 
is taken seriously, $E_{\mu \nu}$ can be the most natural "matter" supporting wormholes.

In this paper we study static, spherically symmetric, asymptotically flat wormhole solutions to the equation $R=0$, where $R$ is the 4-dimensional scalar curvature. Since $E_{\mu \nu}$ has zero trace, $R=0$ is an immediate consequence of (11). $R=0$ is a single equation connecting two metric functions, $\gamma(r)$ and $f(r)$, and can be solved with respect to $f$ for arbitrary $\gamma$. We show that almost any $\gamma(r)$ satisfying some minimal requirements (smoothness and compatibility with asymptotic flatness) gives rise to a family of wormhole solutions with the throat radius as a free parameter. Both symmetric and asymmetric wormholes are obtained. We consider some particular examples, and, in addition to new wormhole metrics, reproduce the results of the recent studies where some solutions with $R=0$ (though under other motivations) were found [31-34]. As a by-product, some black hole solutions and solutions with naked singularities are also obtained. In the brane world framework, there remains a nontrivial problem to be solved: to inscribe the intrinsic brane geometry of the above solutions into the full 5-dimensional picture. Our 4-dimensional wormhole solution can have an arbitrary size of the wormhole throat, but a restriction can quite probably appear from 5-dimensional geometry. Meanwhile, the present class of 4-metrics with zero scalar curvature can be of interest by itself.

The paper is organized as follows. In Sec. 2 we solve the equation $R=0$ and formulate the conditions under which the solution describes a symmetric or asymmetric wormhole; in Sec. 3 we discuss a few particular examples; Sec. 4 contains some observations and concluding remarks.

\section{II. $R=0$ : THE GENERAL SOLUTION}

The general static, spherically symmetric metric in 4 dimensions in the curvature coordinates has the form

$$
d s^{2}=\mathrm{e}^{2 \gamma(r)} d t^{2}-\mathrm{e}^{2 \alpha(r)} d r^{2}-r^{2} d \Omega^{2}
$$

where $d \Omega^{2}=d \theta^{2}+\sin ^{2} \theta d \phi^{2}$ is the linear element on a unit sphere 37 .

The metric (2) gives, according to (11), the following expressions for the components of the effective SET $E_{\mu}^{\nu}$, namely, the energy density $\rho=E_{t}^{t}$, the radial pressure $p_{\text {rad }}=-E_{r}^{r}$ and the lateral pressure $p_{\perp}=-E_{\theta}^{\theta}=-E_{\phi}^{\phi}$ :

$$
\begin{aligned}
-\rho & =\frac{1}{r^{2}}\left(\mathrm{e}^{-2 \alpha}-1\right)-\frac{2 \alpha_{r}}{r} \mathrm{e}^{-2 \alpha} \\
p_{\mathrm{rad}} & =\frac{1}{r^{2}}\left(\mathrm{e}^{-2 \alpha}-1\right)+\frac{2 \gamma_{r}}{r} \mathrm{e}^{-2 \alpha} \\
p_{\perp} & =\mathrm{e}^{-2 \alpha}\left(\gamma_{r r}+\gamma_{r}^{2}-\alpha_{r} \gamma_{r}+\frac{\gamma_{r}-\alpha_{r}}{r}\right)
\end{aligned}
$$

where the subscript $r$ denotes $d / d r$. In case $R=0$ one evidently has $2 p_{\perp}=\rho-p_{\text {rad }}$.
Let us also write down the Kretschmann scalar for the metric (2):

$$
\begin{aligned}
\mathcal{K} & =R_{\mu \nu}^{\rho \sigma} R \rho \sigma^{\mu \nu}=4 K_{1}^{2}+8 K_{2}^{2}+8 K_{3}^{2}+4 K_{4}^{2}, \\
K_{1} & =\mathrm{e}^{-2 \alpha}\left(\gamma_{r r}+\gamma_{r}^{2}-\alpha_{r} \gamma_{r}\right), \quad K_{2}=\mathrm{e}^{-2 \alpha} \frac{\gamma_{r}}{r}, \\
K_{3} & =-\frac{1}{r} \mathrm{e}^{-2 \alpha} \alpha_{r}, \quad K_{4}=\frac{1}{r^{2}}\left(1-\mathrm{e}^{-2 \alpha}\right) .
\end{aligned}
$$

The finiteness of $\mathcal{K}$ is a natural regularity criterion for the geometries to be discussed. Indeed, $\mathcal{K}$ is a sum of squares of all components $R_{\mu \nu}^{\varepsilon \sigma}$ of the Riemann tensor for the metric (2), therefore $\mathcal{K}<\infty$ is necessary and sufficient for finiteness of all algebraic curvature invariants.

The condition $R=0$ which follows from (1) can be written as a linear first-order equation with respect to $f(r) \stackrel{\text { def }}{=} r \mathrm{e}^{-2 \alpha}$ :

$$
f_{r}\left(2+r \gamma_{r}\right)+f\left(2 r \gamma_{r r}+2 r \gamma_{r}^{2}+3 \gamma_{r}\right)=2,
$$

Its general solution is

$$
f(r)=\frac{2 \mathrm{e}^{-2 \gamma+3 \Gamma}}{\left(2+r \gamma_{r}\right)^{2}} \int\left(2+r \gamma_{r}\right) \mathrm{e}^{2 \gamma-3 \Gamma} d r
$$

where

$$
\Gamma(r)=\int \frac{\gamma_{r} d r}{2+r \gamma_{r}}
$$

Thus, choosing the form of $\gamma(r)$ arbitrarily, we obtain $f(r)$ from (8), and, after fixing the integration constant, the metric is known completely at least in the region where $\mathrm{e}^{\gamma}$ and $\mathrm{e}^{\alpha}$ are smooth and nonzero.

Let us now make clear how to choose the function $\gamma(r)$ (the so-called redshift function) and the integration constant in Eq. (8) in order to obtain a wormhole solution. We note for reference purposes that in many papers devoted to wormholes, beginning with [1], the function $\mathrm{e}^{2 \alpha}$ is expressed as $[1-b(r) / r]^{-1}$ where $b(r)$ is the so-called shape function. Our $f(r)$ is then equal to $r-b(r)$.

The coordinate $r$, which proves to be convenient for solving Eq. (7), is not an admissible coordinate in the whole space for wormhole solutions since in this case $r$ has at least one minimum, and the solution in terms of $r$ therefore splits into at least two branches. As an admissible coordinate one can take, e.g., the Gaussian coordinate $l$ (proper length along the radial direction) connected with $r$ by the relation $l=\int \mathrm{e}^{\alpha} d r$, and the metric is rewritten as

$$
d s^{2}=\mathrm{e}^{2 \gamma(l)} d t^{2}-d l^{2}-r^{2}(l) d \Omega^{2} .
$$

We seek static, traversable, twice asymptotically flat wormhole solutions. So we require: (i) there should be two flat asymptotics: $l \in \mathbb{R} ; r \approx|l| \rightarrow \infty$ and $\gamma=$ const $+O\left(r^{-1}\right)$ as $l \rightarrow \pm \infty$; (ii) both functions $r(l)>0$ and $\gamma(l)$ should be smooth (at least $C^{2}$ ) in the whole range $l \in \mathbb{R}$. This guarantees the absence of curvature singularities and horizons (the latter correspond to $\gamma \rightarrow$ 
$-\infty$ which is ruled out). This also means that $r(l)$ should have at least one regular minimum, $r_{\min }>0$ (throat), at some value of $l$. Moreover, returning to functions of $r$, we see that at a flat asymptotic $\mathrm{e}^{\alpha} \rightarrow 1$ and $f(r) \approx r$.

Suppose, without loss of generality, that a minimum of $r(l)$, that is, a wormhole throat, is located at $l=0$. Then $r(0)=r_{0}>0, r_{l}(0)=0$ and (generically) $r_{l l}(0)>0$, where the subscript $l$ denotes $d / d l$. Near $l=0$ one has $r-r_{0} \sim l^{2}$, hence the metric function $\mathrm{e}^{2 \alpha(r)}$ behaves as $\left(r-r_{0}\right)^{-1}$, and $f(r)=r \mathrm{e}^{-2 \alpha} \sim r-r_{0}$. In other words, a simple zero of $f(r)$ is an indicator of a wormhole throat provided $\gamma(r)$ is smooth and finite at the same $r$.

On the other hand, the derivative $\gamma_{l}(0)$ may be zero (which is always the case if the wormhole is symmetric with respect to the throat) or nonzero. If $\gamma_{l}(0)=0$, we shall have $\gamma_{r}\left(r_{0}\right)<\infty$. If, on the contrary, $\gamma_{l}(0) \neq 0$, then near $r_{0}$ we have $\gamma_{r} \sim 1 /|l| \sim \sqrt{r-r_{0}}$, so that

$$
\gamma(r) \approx \gamma\left(r_{0}\right)+k \sqrt{r-r_{0}}, \quad k>0
$$

We cannot put $k<0$ since then we would obtain the expression $2+r \gamma_{r}$ ranging from 2 (at spatial infinity) to $-\infty$ at $r=r_{0}$, so that $2+r \gamma_{r}$ would vanish at some $r>r_{0}$ causing a singularity in (8).

We are now ready to single out a class of symmetric wormhole metrics (W1) and a class of potentially asymmetric wormhole metrics (W2) on the basis of the solution (8).

W1. Specify the function $\gamma(r)$, smooth in the range $r_{0} \leq r<\infty, r_{0}>0$, in such a way that $\gamma(\infty)=0$, $\gamma_{r}\left(r_{0}\right)<\infty$, and $2+r \gamma_{r}>0$ in the whole range. Fix the integration constant in (8) by performing integration from $r_{0}$ to $r$. Then these $\gamma(r)$ and $f(r)$ determine a wormhole which has a throat at $r=r_{0}$ and is symmetric with respect to it.

Indeed, by construction, $f(r) \sim r-r_{0}$ near $r_{0}$. Introducing the new coordinate $x$ by the relation $r=r_{0}+x^{2}$, we have $\mathrm{e}^{2 \alpha} d r^{2} \sim\left(r-r_{0}\right)^{-1} d r^{2}=4 d x^{2}$, which leads to a perfectly regular metric whose all coefficients are even functions of $x \in \mathbb{R}$. Both $x \rightarrow+\infty$ and $x \rightarrow-\infty$ are flat asymptotics.

Each $\gamma(r)$ chosen as prescribed creates a family of symmetric wormholes with zero scalar curvature. The family is parametrized by the throat radius $r_{0}$, taking arbitrary values in the range where $\gamma(r)$ is regular and $2+r \gamma_{r}>0$.

Another procedure is applicable to functions $\gamma(r)$ behaving according to Eq. (11).

W2-a. Specify the function $\gamma(r)$, smooth in the range $r_{0} \leq r<\infty, r_{0}>0$, such that $\gamma(\infty)=0,2+r \gamma_{r}>0$ in the whole range, and Eq. (11) holds near $r_{0}$. Then, for proper values of the integration constant in (8), the sphere $r=r_{0}$ is a wormhole throat, and the solution is smoothly continued beyond it.

Indeed, the solution (8) may be rewritten as follows:

$$
f(r)=\frac{\mathrm{e}^{-2 \gamma+3 \Gamma}}{\left(1+\frac{1}{2} r \gamma_{r}\right)^{2}}\left[\int_{r_{0}}^{r}\left(1+\frac{1}{2} r \gamma_{r}\right) \mathrm{e}^{2 \gamma-3 \Gamma} d r+C\right]
$$

Suppose $C>0$. Then $f(r)$ behaves near $r_{0}$ as $r-r_{0}=$ : $x^{2}$, while $\gamma=\gamma\left(r_{0}\right)+k x+O\left(x^{2}\right)$. The metric smoothly behaves at $r=r_{0}(x=0)$ in terms of the new coordinate $x$ and can be continued through this sphere. One cannot, however, guarantee that this continuation will lead to another flat spatial infinity to yield an asymmetic wormhole, since the further behavior of $\gamma(x)$ and $f(x)$ may lead to a horizon or to a singularity.

If we choose $C \leq 0$ in (12), we obtain two other situations:

W2-b. If $C<0$, then $f\left(r_{0}\right)<0$; recalling that $f \sim r$ at large $r$, we see that $f(r)=0$ at some value $r=r_{1}>r_{0}$, where $\gamma_{r}$ is finite, and we return to the circumstances described as W1, obtaining a symmetric wormhole with $r \geq r_{1}$, and the sphere $r=r_{1}$ is its throat.

W2-c. If $C=0$, then near $r_{0}$ we obtain $f(r) \sim(r-$ $\left.r_{0}\right)^{3 / 2}$, and the metric is regularized at $r=r_{0}$ by another substitution: $r-r_{0}=\xi^{4}$. As a result, Eq. (11) yields

$$
\gamma=\gamma\left(r_{0}\right)+k \xi^{2}+\text { further even powers of } \xi
$$

and we again obtain a symmetric wormhole, but now with a quartic behavior of $r$ near its minimum as a function of the admissible coordinate $\xi \in \mathbb{R}$.

\section{EXAMPLES}

We will present expressions for the metric functions $\gamma$ and $f$, the effective "tidal" energy density $\rho$ and the sum $\rho+p_{\text {rad }}$, which characterizes violation of the null energy condition (for static, spherically symmetric systems this condition reduces to $\left.\rho+p_{\text {rad }} \geq 0\right)$.

We use the time scale of a remote observer at rest and so always assume that $\mathrm{e}^{\gamma} \rightarrow 1$ as $r \rightarrow \infty$.

1. The simplest example is obtained for $\gamma \equiv 0$. Choosing any $r_{0}>0$ and applying the W1 algorithm of Sec. 2, we simply obtain $f(r)=r-r_{0}$. This is a symmetric wormhole solution known as the spatial Schwarzschild geometry 31]:

$$
\begin{aligned}
d s^{2} & =d t^{2}-\left(1-\frac{r}{r_{0}}\right)^{-1} d r^{2}-r^{2} d \Omega^{2} \\
& =d t^{2}-4\left(r_{0}+x^{2}\right) d x^{2}-\left(r_{0}+x^{2}\right)^{2} d \Omega^{2}
\end{aligned}
$$

The effective SET $E_{\mu}^{\nu}$ has the form $T_{\mu}^{\nu}=$ $\operatorname{diag}\left(0,-p_{r}, p_{r} / 2, p_{r} / 2\right)$ with the radial pressure

$$
p_{r}=-r_{0} / r^{3}
$$

2. Our next example uses the Schwarzschild form of $\gamma$ :

$$
\mathrm{e}^{2 \gamma}=1-\frac{2 m}{r}, \quad m>0
$$


Choosing any $r_{0}>2 m$, we obtain according to the W1 prescription:

$$
\begin{aligned}
f(r) & =\frac{(r-2 m)\left(r-r_{0}\right)}{r-3 m / 2} \\
d s^{2} & =\left(1-\frac{2 m}{r}\right) d t^{2}-\frac{\left(1-\frac{3 m}{2 r}\right) d r^{2}}{\left(1-\frac{2 m}{r}\right)\left(1-\frac{r_{0}}{r}\right)}-r^{2} d \Omega^{2} \\
& =\frac{x^{2}+r_{0}-2 m}{r_{0}+x^{2}} d t^{2} \\
- & \frac{4\left(r_{0}+x^{2}\right)\left(r_{0}+x^{2}-\frac{3}{2} m\right)}{x^{2}+r_{0}-2 m} d x^{2}-\left(x^{2}+r_{0}\right)^{2} d \Omega^{2}
\end{aligned}
$$

This is evidently a symmetric wormhole geometry for any $r_{0}>2 m \geq 0$, or for any $r_{0}>0$ in case $m<0$. The Schwarzschild metric is restored from (17) in the special case $r_{0}=3 \mathrm{~m} / 2$.

The SET components of interest are

$$
\begin{aligned}
\rho & =\frac{m\left(r_{0}-\frac{3}{2} m\right)}{2 r^{2}\left(r-\frac{3}{2} m\right)^{2}} ; \\
\rho+p_{\text {rad }} & =-\frac{(r-2 m)\left(r_{0}-\frac{3}{2} m\right)}{r^{2}\left(r-\frac{3}{2} m\right)^{2}} .
\end{aligned}
$$

The metric (17) was obtained by Casadio, Fabbri and Mazzacurati [33] in search for new brane-world black holes and by Germani and Maartens [34] as a possible external metric of a homogeneous star on the brane, but the existence of traversable wormhole solutions for $r_{0}>2 m$ (in the present notations) was not mentioned. It was supposed in 33 that the post-Newtonian parameters of the metric must be close to their Einstein values for experimental reasons and therefore restricted their study to configurations close to Schwarzschild. Then $r_{0}$ must be close to $3 \mathrm{~m} / 2$. In this case, as in the Schwarzschild metric, $r=2 m$ is an event horizon, but, according to [33], the space-time structure depends on the sign of $\eta=r_{0}-3 m / 2$. If $\eta<0$, the structure is that of a Schwarzschild black hole, but the curvature singularity is located at $r=3 \mathrm{~m} / 2$ instead of $r=0$. If $\eta>0$, the solution describes a nonsingular black hole with a wormhole throat at $r=r_{0}$ inside the horizon, in other words, a non-traversable wormhole 33].

We would here remark that, in our view, such hypothetic objects as brane-world black holes or wormholes, not necessarily of astrophysical size, need not necessarily conform to the restrictions on the post-Newtonian parameters obtained from the Solar system and binary pulsar observations, and it therefore makes sense to discuss the full range of parameters which are present in the solutions.

3. Consider the extreme Reissner-Nordström form of $\gamma(r)$ :

$$
\mathrm{e}^{2 \gamma}=\left(1-\frac{2 m}{r}\right)^{2}, \quad m>0
$$

The W1 procedure now leads to

$$
\begin{aligned}
& f(r)=\frac{\left(r-r_{0}\right)\left(r-r_{1}\right)}{r}, \quad r_{1} \stackrel{\text { def }}{=} \frac{m r_{0}}{r_{0}-m} ; \\
& d s^{2}=\left(1-\frac{2 m}{r}\right)^{2} d t^{2}-\frac{r^{2} d r^{2}}{\left(r-r_{0}\right)\left(r-r_{1}\right)}-r^{2} d \Omega^{2} \\
= & \left(1-\frac{2 m}{r_{0}+x^{2}}\right)^{2} d t^{2}-4 \frac{\left(r_{0}+x^{2}\right)^{2} d x^{2}}{r_{0}-r_{1}+x^{2}}-\left(r_{0}+x^{2}\right) d \Omega^{2} .
\end{aligned}
$$

where we assume $r_{0}>2 m$, so that $r_{1}<r_{0}$. This is a symmetric wormhole metric. The SET components of interest are

$$
\begin{aligned}
\rho & =\frac{m r_{0}^{2}}{r^{4}\left(r_{0}-m\right)}, \\
\rho+p_{\text {rad }} & =-\frac{\left(r_{0}-2 m\right)^{2}}{\left.r^{2}(r-2 m)\left(r_{0}-m\right)\right)} .
\end{aligned}
$$

In the solution (20), $r_{0}$ may be regarded as an integration constant, so it is of interest what happens if $r_{0} \leq 2 m$. Evidently, $r_{0}=2 m$ leads to the extreme Reissner-Nordström black hole metric (which is well known to possess a zero Ricci scalar, as does the general Reissner-Nordström metric). In case $2 m>r_{0}>m$, we have $r_{1}>2 m$, and we again obtain a symmetric wormhole, but now $r$ ranges from $r_{1}$ to infinity and $r=r_{1}$ is the throat. Actually, $r_{0}$ and $r_{1}$ exchange their roles as compared with the case $r_{0}>2 m$. This property was expected due to symmetry between $r_{0}$ and $r_{1}$ in the metric (21).

The value $r_{0}=m$ is meaningless. Lastly, $r_{0}<m$ leads either to $r_{1}<0$ (for $r_{0} \geq 0$ ) or to $0<r_{1}<2 m$ (for $\left.r_{0}<0\right)$. The solution exists in both cases for $r>2 m$ only, and $r=2 m$ turns out to be a naked singularity, as is confirmed by calculating the Kretschmann scalar.

4. Consider an example belonging to class W2 described in the previous section. Namely, let us choose

$$
\mathrm{e}^{2 \gamma}=(1-b+b \sqrt{1-2 m / r})^{2}
$$

with $b=$ const $\neq 0$. The special cases $b=0$ and $b=1$ nave been already discussed in Examples 1 and 2, respectively. The form (23) of $\mathrm{e}^{\gamma(r)}$ has been found [31, 32, 33] by solving the equation $R=0$ under the condition that the energy density $T_{0}^{0}$ is zero, whence it followed that $\mathrm{e}^{-2 \alpha}=1-2 m / r$, and, in our notation, $f(r)=r-2 m$. Note that the Schwarzschild mass, found from the large $r$ behavior of $\gamma(r)$, is equal to $b m$ rather than $m$.

Knowing that $f(r)=r-2 m$ is a special solution to the inhomogeneous equation (7) with $\gamma(r)$ given by (23), we can make easier the integration in (8) by writing the solution as $f(r)=2 r-m+f_{1}(r)$ where $f_{1}$ is a general solution to the corresponding homogeneous equation. We obtain

$$
f(r)=r-2 m+C \frac{\mathrm{e}^{-2 \gamma+3 \Gamma}}{\left(2+r \gamma_{r}\right)^{2}},
$$


where $C_{1}=$ const and $\Gamma$ has been defined in (9). The form of $\Gamma(r)$ depends on the constant $c \stackrel{\text { def }}{=} 2(1-b) / b$ (the case $b=0$ is excluded):

$$
\begin{aligned}
& \mathrm{e}^{3 \Gamma}= \\
& \begin{cases}\left(1+2 c v+3 v^{2}\right) \exp \left[-\frac{2 c}{c^{\prime}} \arctan \frac{c+3 v}{c^{\prime}}\right], & c<\sqrt{3} \\
(1+\sqrt{3} v)^{2} \exp [2 /(1+\sqrt{3} v)] & c=\sqrt{3} \\
\left(1+2 c v+3 v^{2}\right)\left[\frac{c+3 v+c^{\prime}}{c+3 v-c^{\prime}}\right]^{-c / c^{\prime}}, & c>\sqrt{3}\end{cases}
\end{aligned}
$$

where we have denoted

$$
c^{\prime}=\sqrt{\left|c^{2}-3\right|}, \quad v=\sqrt{1-\frac{2 m}{r}} .
$$

For all three cases in (25), depending on the integration constant $C_{1}$, one can single out the behaviors of classes W2-a, W2-b and W2-c (in their description in Sec. 2 one should substitute $\left.r_{0}=2 m\right)$. The critical value of $C_{1}$, corresponding to $C=0$ in Eq. (12), is

$$
C_{1 \mathrm{cr}}=-\left.2 m \mathrm{e}^{-3 \Gamma}\right|_{v=0} .
$$

It corresponds to integration in Eq. (8) from $r_{0}$ to $r$, and the solution then belongs to class W2-c, a symmetric wormhole with a quartic dependence of $r$ on the admissible coordinate $\xi$.

The case $C_{1}<C_{1 \mathrm{cr}}$ corresponds to integration in (8) from some $C_{1}$-dependent radius $r_{1}>2 m$ to $r$, and the solution belongs to type W2-b equivalent to W1: a symmetric wormhole with an arbitrary throat radius $r_{1}>2 \mathrm{~m}$. We will not write down the full cumbersome expressions for the metric for $C_{1} \leq C_{1 \mathrm{cr}}$ since the qualitative properties of the solutions are already clear.

Of greater interest are solutions with $C_{1}>C_{1 \mathrm{cr}}$, for which $r=2 m$ is an asymmetric throat [class W2-a]. This is the only class in which the metric continued beyond the throat behaves "individually", i.e., depends on the specific choice of $\gamma(r)$ and $C_{1}$ rather than follows the above general description.

A good coordinate for passing the throat $r=2 m$, more convenient than the previously used coordinate $x$, is $v$ defined in (26). We have $r(v)=2 m /\left(1-v^{2}\right)$; the original spatial asymptotic corresponds to $v=1$, the throat is located at $v=0$, and another spatial asymptotic can be located at $v=-1$ if the metric avoids singularities on the way to it. Let us find out whether it is the case.

The requirement $\mathrm{e}^{\gamma}>0$ at $v \geq-1$ leads to $b<1 / 2$, hence $c>2$, and we are left with the third line in the expression (25) for $\Gamma(v)$. Then the metric coefficient $g_{v v}$ is given by

$$
\begin{aligned}
&-g_{v v}=\frac{16 m^{2}}{\left(1-v^{2}\right)^{2}} \\
& \times\left[1+C_{1} \frac{1-v^{2}}{6 m}\left(v-v_{-}\right)^{3 v_{-} / c^{\prime}}\left(v-v_{+}\right)^{-3 v_{+} / c^{\prime}}\right]^{-1}
\end{aligned}
$$

where $v_{ \pm}$are roots of the trinomial $P(v)=3 v^{2}+2 c v+1$ :

$$
v_{ \pm}=\frac{1}{3}\left(-c \pm \sqrt{c^{2}-3}\right)=\frac{1}{3}\left(-c \pm c^{\prime}\right) .
$$

For $c>2$, we have $v_{-}<-1$ whereas the other root $v_{+}$lies between 0 and -1 . On the other hand, $-3 v_{+} / c^{\prime}$, i.e., the exponent of the binomial $\left(v-v_{+}\right)$in (28), is a number between 0 and 1 . Therefore $g_{v v}$ is finite at $v=v_{+}$but has an infinite derivative with respect to $v$. Transforming back to $r$ (for $v<0$, the transformation is $v=-\sqrt{1-2 m / r})$, we observe that the metric coefficient $g_{r r}=-e^{2 \alpha}\left[\right.$ see (2)] is singular at $r=r_{+}=2 \mathrm{~m} /(1-$ $\left.v_{+}\right)^{2}$. More precisely, $\mathrm{e}^{2 \alpha}$ is finite but contains a term proportional to $\left(r-r_{+}\right)^{k}$ where $0<k<1$, hence $\alpha_{r} \sim$ $\left(r-r_{+}\right)^{k-1} \rightarrow \infty$, and the Kretschman scalar (6) blows up due to the divergence of its constituents $K_{1}$ and $K_{3}$.

We conclude that, under the choice (23) of $\gamma(r)$, the class of solutions (W2-a) does not contain wormhole solutions. Having passed the throat at $r=2 m(v=0)$, we ultimately arrive at a singularity or maybe a horizon, which is not excluded in case $b>1 / 2$.

An exception is the case $C_{1}=0$, when we return to the solution known from Refs. [31, 32, 33], which has been described at length in these papers. We will only mention the main points in our notations. The metric in terms of $v$ is

$$
d s^{2}=(1-b+b v)^{2} d t^{2}-\frac{16 m^{2}}{\left(1-v^{2}\right)^{4}} d v^{2}-\frac{4 m^{2}}{\left(1-v^{2}\right)^{2}} d \Omega^{2} .
$$

In case $b=1$ it is another form of the Schwarzschild metric. For $b>1 / 2$ but $b \neq 1$, the sphere $v=(b-1) / b$ is a naked singularity, as may be concluded from the fact that $\gamma_{r} \rightarrow \infty$ while $\alpha$ and $r$ are finite, hence the quantity $K_{2}$ in (6) blows up. This singularity is located at positive $v$, i.e., before reaching the throat $v=0$, if $b>1$ and at negative $v$, beyond the throat, if $b<1$. Note that for $v<0$ we have in the curvature coordinates $g_{t t}=\mathrm{e}^{2 \gamma}=$ $1-b-b \sqrt{1-2 m / r}$.

In case $b<1 / 2$ the metric (29) describes an asymmetric wormhole even having different signs of mass at its two flat asymptotics: the mass is equal to $b m$ at $v=1$ and to $-b m /(1-2 b)$ at $v=-1$.

Lastly, if $b=1 / 2$, then $v=-1$ is a horizon having an infinite area and zero Hawking temperature, like the previously described cold black holes in scalar-tensor theories of gravity [9]. The spatial part of the metric is flat at $v \rightarrow-1$. Moreover, as is directly verified, the canonical parameter for timelike, spacelike or null geodesics takes an infinite value at at $v=1$, which means that this space-time is geodesically complete (as are wormhole space-times), and no further continuation is required.

\section{CONCLUDING REMARKS}

We have seen that the equation $R=0$ leads to a great number of wormhole solutions. Symmetric wormhole so- 
lutions of class W1 can be obtained from any $\gamma(r)$ providing asymptotic flatness; asymmetric wormhole solutions belonging to class W2-a require somewhat more special conditions. As follows from Examples 1-3, wormholes are not always connected with negative (effective) energy densities $\rho$; they can appear with $\rho>0$, but only with comparatively large negative pressures maintaining violation of the null energy condition. Example 3 shows that, for given $\gamma(r)$, sometimes even more wormhole solutions can be obtained than was expected in search for class W1 solutions. Example 4 shows that asymmetric wormholes are more difficult to obtain from the general solution (8) than symmetric ones.

Black-hole solutions can also be obtained from (8) but under more restrictive conditions. Indeed, given a specific function $\mathrm{e}^{\gamma(r)}$ increasing from zero at some $r=r_{h}$ to 1 at $r=\infty$, a wormhole solution to $R=0$ can be obtained with a throat at any $r>r_{h}$ whereas in a black hole solution the event horizon is fixed at $r=r_{h}$. Positive functions $\mathrm{e}^{\gamma(r)}$ lead to numerous wormhole solutions but not black hole ones. Thus, roughly speaking, wormholes as solutions to $R=0$ are more numerous than black holes.

All this referred to metrics satisfying the condition $R=0$ in 4 dimensions, which admits an interpretation as the brane metric. It has been claimed that "any 4-dimensional space-time with $R=0$ gives rise to a 3-brane world without surface stresses embedded in a 5-dimensional space-time" 35] since the embedding contains a very significant arbitrariness. Never- theless, a complete model requires knowledge of the full 5-dimensional space-time. In other words, one should "evolve" the 4-metric into the bulk, using this 4-matric as initial data for the 5-dimensional equations. It is rather a difficult task, as was demonstrated in a study of particular black hole solutions in Refs. [30, 36]. There are, however, two favorable circumstances. One is the wealth of wormhole solutions: there is actually an arbitrary function $\gamma(r)$ leading to wormholes on the brane, which must in turn lead to a wide choice of suitable bulk functions. The other is the global regularity of wormhole space-times, and one can expect that the bulk incorporating them will also be regular. (It may be recalled that it was the singular nature of black hole solutions that caused some technical difficulties in Ref. [30].) We hope that it will be possible to obtain meaningful complete wormhole models within the brane world concept; the work is in progress.

\section{Acknowledgment}

The work was supported in part by grant No. R012000-000-00015-0 from the Korea Science and Engineering Foundation and in part by Asia Pacific Center for Theoretical Physics. KB is grateful to the colleagues from Ewha Womans University, Seoul, for kind hospitality during his stay in October-November 2002.
[1] M.S. Morris and K.S. Thorne, Am. J. Phys. 56, 395 (1988);

M.S. Morris, K.S. Thorne and U. Yurtsever, Phys. Rev. Lett. 61, 1449 (1988).

[2] M. Visser, "Lorentzian Wormholes: from Einstein to Hawking" (AIP, Woodbury, 1995).

[3] M. Visser and D. Hochberg, "Geometric wormhole throats", gr-qc/9710001.

[4] D. Hochberg and M. Visser, Phys. Rev. D 58, 044021 (1998); gr-qc/9802046.

[5] H. Ellis, J. Math. Phys. 14, 104 (1973).

[6] K.A. Bronnikov, Acta Phys. Polon. B4, 251-273 (1973).

[7] T. Kodama, Phys. Rev. D 18, 3529 (1978).

[8] A.G. Agnese and M. La Camera, Phys. Rev. D 51, 2011 (1995).

[9] K.A. Bronnikov, G. Clément, C.P. Constantinidis and J.C. Fabris, Phys. Lett. 243A, 121 (1998), grqc/9801050; Grav. \& Cosmol. 4, 128 (1998), grqc/9804064.

[10] S.-W. Kim and S.P. Kim, Phys. Rev. D 58, 087703 (1998).

[11] S.-W. Kim and H. Lee, Phys. Rev. D 63, 64014 (2001).

[12] S.A. Hayward, Phys. Rev. D 65, 124016 (2002); grqc/0202059

[13] C. Armendáriz-Picón, gr-qc/0201027.

[14] C. Barceló and M. Visser, Phys. Lett. 466B, 127-134 (1999); Class. Qu. Grav. 17, 38432000.
[15] S.V. Sushkov and S.-W. Kim, Class. Qu. Grav. 19, 4909 (2002).

[16] K.A. Bronnikov, J. Math. Phys. 43, No. 12 (2002), grqc/0204001

[17] K.A. Bronnikov and S.V. Grinyok, Grav. 85 Cosmol. 7, 297 (2001), gr-qc/0201083; Festschrift in honour of Prof. Mario Novello, 2002; gr-qc/0205131.

[18] W. Graf, gr-qc/0209002.

[19] L.A. Anchordoqui, A.G. Grunfeld and D.F. Torres, grqc/9707025, Grav. $\&$ Cosmol. 4, 287 (1998).

[20] D. Hochberg, A. Popov and S. Sushkov, Phys. Rev. Lett. 78, 2050 (1997).

[21] B.E. Taylor, W.A. Hiscock and P.R. Anderson, Phys. Rev. D 55, 6116 (1997).

[22] V. Khatsymovsky, Phys. Lett. 429B, 254 (1998).

[23] S. Nojiri, O. Obregon, S.D. Odintsov and K.E. Osetrin, Phys. Lett. 449B, 173 (1999); 458B, 19 (1999).

[24] L. Randall and R. Sundrum, Phys. Rev. Lett. 83, 3370 (1999).

[25] L. Randall and R. Sundrum, Phys. Rev. Lett. 83, 4690 (1999).

[26] V.A. Rubakov, "Large and infinite extra dimensions", Phys. Usp. 44, 871 (2001); hep-ph/0104152;

R. Maartens, "Geometry and dynamics of the brane world", gr-qc/0101059;

D. Langlois, "Gravitation and cosmology in a brane universe", gr-qc/0207047. 
[27] P. Horava and E. Witten, Nucl. Phys. B 460, 506 (1996); ibid., 475, 94 (1996).

[28] T. Shiromizu, K. Maeda and M. Sasaki, Phys. Rev. D 62, 024012 (2000).

[29] D. Vollick, Gen. Rel. Grav. 34, 1 (2002).

[30] A. Chamblin, H.S. Reall, H. Shinkai and T. Shiromizu, Phys. Rev. D 63, 064015 (2001); hep-th/0008177.

[31] N. Dadhich, S. Kar, S. Mukherjee and M. Visser, Phys. Rev. D 65, 064004 (2002).

[32] A.G. Agnese and M. La Camera, gr-qc/0203067.

[33] R. Casadio, A. Fabbri and L. Mazzacurati, Phys. Rev. D
65, 084040 (2001); gr-qc/0111072.

[34] C. Germani and R. Maartens, Phys. Rev. D 64, 124010 (2001); hep-th/0107011.

[35] D. Vollick, Gen. Rel. Grav. 34, 471 (2002).

[36] R. Casadio and L. Mazzacurati, "Bulk shape of braneworld black holes", gr-qc/0205129.

[37] The sign conventions are: the metric signature (+- - ); the curvature tensor $R_{\mu \rho \nu}^{\sigma}=\partial_{\nu} \Gamma_{\mu \rho}^{\sigma}-\ldots$, so that, e.g., the Ricci scalar $R>0$ for de Sitter space-time; and the stress-energy tensor such that $T_{t}^{t}$ is the energy density. 